\title{
Implizite Einstellungen von Kindern im Grundschulalter gegenüber Menschen mit Migrationshintergrund
}

\author{
Justine Stang (D, Sabrina König und Nele McElvany \\ Institut für Schulentwicklungsforschung, Technische Universität Dortmund, Deutschland
}

\begin{abstract}
Zusammenfassung: Erwachsene und Jugendliche ohne Migrationshintergrund (MIG) schreiben Personen mit türkischem MIG, der größten Migrationsgruppe in Deutschland, geringere Kompetenzen und Leistungen zu. Inwiefern bereits bei Kindern solche (negativen) impliziten Einstellungen gegenüber Menschen mit türkischem MIG vorliegen, ist bislang ungeklärt und wurde daher in dieser Studie überprüft. Auch wurde analysiert, ob sich Grundschulkinder mit türkischem und ohne MIG in ihren Einstellungen unterscheiden. Zudem wurde geprüft, ob die soziale Integration, die wahrgenommene Diskriminierung sowie die Identifikation mit der Aufnahme- und Herkunftskultur mit impliziten Einstellungen in Zusammenhang standen. Datengrundlage bildeten Angaben von 240 Viertklässlerinnen und -klässlern (44.6\% weiblich, $18.3 \%$ mit türkischem MIG). Zur Messung impliziter Einstellungen bearbeiteten die Kinder einen Impliziten Assoziationstest. Des Weiteren beantworteten sie Fragen zu den interessierenden Variablen. Bei den Grundschulkindern lagen negative implizite Einstellungen vor $(d=0.21)$. Konkret verfügten Kinder ohne MIG im Gegensatz zu Kindern mit türkischem MIG im Mittel über negative implizite Einstellungen $(d=0.39)$. Für die Gesamtstichprobe zeigte sich, dass eine stärkere Identifikation mit Deutschland ( $B=.14)$, der Aufnahmekultur, in Zusammenhang mit negativen impliziten Einstellungen stand $\left(R^{2}=.03\right)$. Die Ergebnisse werden sowohl inhaltlich als auch hinsichtlich methodischer Gesichtspunkte diskutiert. Implikationen für Forschung und Praxis werden vorgestellt.
\end{abstract}

Schlüsselwörter: Grundschule, Einstellungen, Impliziter Assoziationstest (IAT), Migrationshintergrund, Stereotype

Implicit attitudes of elementary school students towards people with migrant background

\begin{abstract}
Adults and young people without a migrant background attribute lower competencies and performances to persons with a Turkish migrant background, the largest migrant group in Germany. To what extent such (negative) implicit attitudes are already present among elementary school children in the fourth grade is unclear and was tested within this study. Furthermore, it was examined whether children with a Turkish migrant background and without a migrant background differ in their attitudes. It was also analyzed whether the variables social integration, perceived discrimination and identification with country of residence as well as the country of origin were related with the extent of implicit attitudes. Data came from 240 fourth graders ( $44.6 \%$ female, $18.3 \%$ with a Turkish migrant background). To measure implicit attitudes, the children worked on an implicit association test. Furthermore, they answered questions about variables of interest. It turned out that fourthgraders hold negative implicit attitudes $(d=0.21)$. In contrast to children with a Turkish migrant background, children without a migrant background had on average negative implicit attitudes $(d=0.39)$. For the whole sample it was found that a higher identification with Germany ( $(B=.14)$, the country of residence, was associated with negative implicit attitudes $\left(R^{2}=.03\right)$. The results are discussed in terms of content and methodological aspects. Implications for research and practice are presented.
\end{abstract}

Keywords: Elementary School, Implicit Association Test (IAT), Implicit Attitudes, Migrant Background, Stereotypes

Schulklassen in Deutschland sind von Heterogenität geprägt (Stanat, Schipolowski, Rjosk, Weirich \& Haag, 2017). Im Jahr 2016 wiesen $33.0 \%$ der gesamten Schülerschaft einen Migrationshintergrund (MIG) auf. Schülerinnen und Schüler mit einem türkischen MIG stellten mit 6.3\% die größte Herkunftsgruppe nichtdeutschstämmiger Kinder und Jugendlicher dar (Statistisches Bundesamt [Destatis]/Wissenschaftszentrum Berlin für Sozialforschung [WZB], 2018). In Studien wurden für Kinder mit türkischem MIG im Mittel im Vergleich zu Kindern ohne MIG geringere sprachliche Kompetenzen berichtet (z.B. Wortschatz: Kigel, McElvany \& Becker, 2015). Als eine mögliche Erklärung für die genannten und weitere leistungsbezogene Disparitäten werden neben der sozialen Herkunft ebenso Einstellungen und Stereotype sowie die damit verbundene Stereotypenbedrohung (Stereotype threat; ST) herangezogen (Sander, Ohle-Peters, McElvany, Zander \& Hannover, 2018; Steele \& Aronson, 1995).

ST beschreibt die Situation, in der das Wissen um ein negatives Stereotyp über eine Gruppe (z. B. , Mädchen sind schlechter in Mathematik als Jungen"), der man angehört, die wahrgenommene Bedrohung auslöst, dieses Stereotyp selbst zu bestätigen. Diese Bedrohung führt bei ansonsten gleichen Leistungsvoraussetzungen $\mathrm{zu}$ Leistungseinbußen, wodurch das negative Stereotyp bestätigt wird (Ap- 
pel, Weber \& Kronberger, 2015; Steele \& Aronson, 1995). ST kann sich nur dann auf eine Leistungs- oder Lernsituation auswirken, wenn das entsprechende negative Stereotyp den Kindern bekannt ist und implizit oder explizit aktiviert wird (z.B. Wasserberg, 2014).

In der Forschungsliteratur zeigte sich, dass Lehrkräfte teilweise negative implizite Einstellungen gegenüber Kindern einer ethnischen Minderheit haben können. Ebenso können Jugendliche in Deutschland negative Einstellungen gegenüber Menschen mit MIG haben und ihnen geringere Kompetenzen in verschiedenen Domänen zuschreiben. Dabei bestehen interindividuelle Unterschiede in der Ausprägung von Einstellungen gegenüber Menschen mit MIG (Glock \& Klapproth, 2017; Martiny, Mok, Deaux \& Froehlich, 2014; Zander, Webster \& Hannover, 2014).

Weiterhin ergaben Studien, dass bereits bei Grundschulkindern Stereotype insbesondere in Bezug auf Geschlecht sowie ST-Effekte zu finden sind (z.B. Hermann \& Vollmeyer, 2016; Tomasetto, Alparone \& Cadinu, 2011). Hinsichtlich (negativer) Einstellungen zu verschiedenen Ethnien wiesen Studien im nicht deutschsprachigen Raum darauf hin, dass auch bereits Kinder zum Beispiel über negative verhaltensbezogene Einstellungen gegenüber Fremdgruppen verfügen können (z.B. Baron \& Banaji, 2006; Bar-Tal, 1996). Unklar ist, ob auch in der deutschen Gesellschaft schon Grundschulkinder negative Einstellungen gegenüber Menschen mit türkischem MIG haben und welche Variablen gegebenenfalls in einem Zusammenhang mit (negativen) Einstellungen stehen.

Aufgrund der weitreichenden Bedeutung von negativen impliziten Einstellungen, die sich zum Beispiel in der Beeinflussung des eigenen, spontanen Verhaltens gegenüber anderen äußern kann (z. B. Perugini, 2005), wurde in dieser Studie untersucht, ob bei Viertklässlerinnen und Viertklässlern implizite Einstellungen gegenüber Menschen mit einem türkischen MIG vorliegen und ob interindividuelle Unterschiede in Einstellungen bestehen. Im schulischen Kontext und bezugnehmend auf Menschen mit MIG sind vor dem Hintergrund theoretischer Überlegungen und empirischer Befunde Konstrukte wie soziale Integration, wahrgenommene Diskriminierung sowie die Identifikation mit der Aufnahme- und Herkunftskultur von Interesse. Daher wurde in dieser Studie analysiert, ob diese Variablen in einem Zusammenhang mit impliziten Einstellungen stehen.

\section{Theoretischer Hintergrund}

\section{Einstellungen}

Einstellungen werden als im Gedächtnis vorhandene Assoziationen zwischen einem Einstellungsobjekt und der
Bewertung dieses Objektes verstanden und können anhand ihrer Valenz (positiv oder negativ), ihrer Intensität (stark oder schwach) und ihrer Art (implizit oder explizit) unterschieden werden (Fazio, 1990; Maio, Haddock \& Verplanken, 2019). Explizite Einstellungen sind bewusste und verbalisierbare Bewertungen. Im Gegensatz dazu handelt es sich bei impliziten Einstellungen um Bewertungen, die automatisch aktiviert werden können und dem Bewusstsein in der Regel nicht zugänglich sind (Dovidio, Hewstone, Glick \& Esses, 2010).

Da Einstellungen als eine psychologische Tendenz verstanden werden, die durch die Beurteilung einer bestimmten Entität entsteht und mit einem gewissen Grad von Gefallen oder Ablehnung ausgedrückt werden kann, können sie in eine kognitive, affektive und behaviorale Komponente unterteilt werden. Bewertungen, Überzeugungen und zugeschriebene Eigenschaften in Bezug auf ein bestimmtes Einstellungsobjekt bilden die kognitive Einstellungsebene. Diese Assoziationen gegenüber dem Einstellungsobjekt werden als Stereotype bezeichnet. Die affektive Komponente äußert sich in einer emotionalen Reaktion, die stärker durch Wertvorstellungen und moralische Überzeugungen charakterisiert ist. Wenn die affektive Komponente gegenüber einem Einstellungsobjekt oder einer Gruppe von Personen negativ ist, wird von einem Vorurteil gesprochen. Die behaviorale Komponente umfasst bestimmte Handlungen gegenüber dem Einstellungsobjekt (Eagly \& Chaiken, 1995; Maio et al., 2019; Zanna \& Rempel, 2008). In dieser Arbeit steht die kognitive Komponente im Fokus.

Die kognitive Komponente kann als abstrakte Wissensstruktur verstanden werden, die eine soziale Gruppe mit einer Reihe von Eigenschaften, Merkmalen und charakteristischen Verhaltensweisen verbindet. Stereotype basieren auf Kategorisierungsprozessen und können automatisch aktiviert werden, sodass die Person sich dessen nicht bewusst sein muss (Dovidio et al., 2010; Kite \& Whitley Jr., 2016). Jedoch kann zum Beispiel kognitive Belastung die Wahrscheinlichkeit reduzieren, dass ein Stereotyp automatisch aktiviert wird (z. B. Gilbert \& Hixon, 1991). Stereotype gegenüber einer Gruppe und ihren Mitgliedern beeinflussen systematisch, wie über diese Gruppe gedacht und auf sie reagiert wird (Dovidio et al., 2010).

Inwiefern Stereotype mit der Realität übereinstimmen, wird im Rahmen der Stereotype accuracy-Forschung untersucht (z.B. Jussim, 2012). Jussim, Crawford und Rubinstein (2015) zeigten in ihrem Review auf, dass die Übereinstimmungen von Stereotyp und Wirklichkeit mittel bis hoch zum Beispiel in Bezug auf Ethnie und Geschlecht ausfallen können. Zur Messung der Stereotypengenauigkeit müssen persönliche Überzeugungen über eine Gruppe erfasst (z.B. „Wie hoch ist der Anteil an Personen mit türkischem MIG in der Schülerschaft?") und mit passen- 
den Kriterien (z.B. statistische Kennzahlen) verglichen werden. Die Stereotypengenauigkeit ist zum Beispiel abhängig von der Repräsentativität des Kriteriums (z.B. Selbstbericht vs. statistische Kennzahlen).

\section{Einstellungen zu Menschen mit Migrationshintergrund}

Implizite Einstellungen können sich bei Kindern zwischen dem zweiten und fünften Lebensjahr entwickeln (z.B. Kite \& Whitley Jr., 2016). Kognitive Fähigkeiten und das Bewusstsein entwickeln sich mit zunehmendem Alter dahingehend weiter, dass Kategorisierungsprozesse nicht mehr nur auf perzeptuellen Gemeinsamkeiten und Unterschieden beruhen, sondern aufgrund der Entwicklung des konzeptuellen Verstehens auch auf internalen, abstrakten Eigenschaften wie zum Beispiel der Ethnizität basieren können (Aboud, 2003; Kite \& Whitley Jr., 2016). Das Vorhandensein von Einstellungen in Bezug auf Geschlecht und Alter konnte bei Kindern mehrfach empirisch gezeigt werden (Bigler \& Liben, 2006; Galdi, Cadinu \& Tomasetto, 2014; Martin \& Ruble, 2010). Hinsichtlich der Einstellungen zu Ethnien ergab eine Arbeit von Baron und Banaji (2006), dass bei sechs- und zehnjährigen Kindern ein proWhite beziehungsweise anti-Black Bias vorlag, der eine Präferenz gegenüber White Americans respektive negative implizite Einstellungen gegenüber African Americans indiziert. Bar-Tal (1996) konnte aufzeigen, dass bei jüdischen Kindergartenkindern in Israel negative verhaltensbezogene Stereotype gegenüber der arabischen Minderheit vorlagen. Rutland (1999) untersuchte 329 Kinder (6-, 8-, 10-, 12-, 14- und 16-Jährige) aus Grund- und Sekundarschulen in Großbritannien und fand heraus, dass die Eigengruppe favorisiert wurde (Briten) und Vorurteile gegenüber einer Fremdgruppe (Deutschen) auftraten, die sich in nicht Mögen äußerten.

Mit Blick auf Einstellungen gegenüber Menschen mit einem türkischen MIG zeigten Studien auf, dass negative Einstellungen bestehen. So fand Asbrock (2010) mittels eines Fragebogens, der die Dimensionen Warmherzigkeit und Kompetenz erfasste, heraus, dass Studentinnen und Studenten Personen mit einem türkischen MIG im Mittel mit geringen Werten auf beiden Dimensionen beurteilten. Auch in Bezug auf Jugendliche ergab sich, dass negative Einstellungen präsent sind: Sowohl türkisch- als auch deutschstämmige Neuntklässlerinnen und Neuntklässler dreier Realschulen waren der Meinung, dass die meisten Menschen die Kompetenzen von Deutschen in Mathematik für höher hielten als die Kompetenzen von Personen mit türkischem MIG (Martiny et al., 2014). Eine OnlineStudie (Schmidt-Daffy, Zander, Sander, McElvany \& Hannover, 2016) brachte hervor, dass deutsche sowie Schüle- rinnen und Schüler mit einem anderen als türkischen MIG im Alter von rund 14 Jahren im Mittel übereinstimmend annahmen, dass deutsche Schülerinnen und Schüler türkischstämmige Schülerinnen und Schüler für weniger kompetent hielten als umgekehrt. Türkischstämmige Schülerinnen und Schüler nahmen deutsche Schülerinnen und Schüler dagegen eher als positiv wahr und meinten, dass sie auch von deutschen Schülerinnen und Schülern als positiv wahrgenommen werden würden. Eine weitere Studie erbrachte, dass die Kompetenzen und Leistungen in der Domäne Mathematik von Menschen mit MIG sowohl von Neuntklässlerinnen und Neuntklässlern mit als auch ohne MIG im Mittel niedriger eingeschätzt wurden (Zander et al., 2014).

\section{Erklärungsmöglichkeiten impliziter Einstellungen}

Eine große Anzahl an Erklärungsmöglichkeiten beziehungsweise Einflussfaktoren, die Einstellungen formen können, werden diskutiert (Maio et al., 2019). Ein Grund dafür, dass sich zum Beispiel Menschen mit und ohne MIG in ihren Einstellungen unterscheiden, stellt die jeweilige Gruppenzugehörigkeit dar. Die Ingroup/Outgroup-Favorisierung (Tajfel, 1982) beschreibt, wie basale Unterscheidungsmerkmale von Gruppen (z. B. Geschlecht) zu Vorurteilen, Stereotypen und Diskriminierung gegenüber der Gruppe, der man nicht angehört (Fremdgruppe), führen können. Der Eigengruppe bestehen gegenüber vornehmlich positive Einstellungen. Im Gegensatz dazu können sich gegenüber der Fremdgruppe negative Einstellungen entwickeln (Brewer, 1999; Grundannahme der Social Identity Theory: Tajfel \& Turner, 1986). So fand zum Beispiel Rutland (1999), dass bei britischen Kindern ab einem Alter von zehn Jahren Präferenzen für die Eigengruppe vorlagen. Im Rahmen von US-amerikanischen Arbeiten zeigte sich etwa, dass White Americans im Mittel stärker positive implizite Präferenzen für die eigene Gruppe aufwiesen und ein negativer Bias in Bezug auf African Americans vorlag (Dasgupta \& Greenwald, 2001; Nosek, Banaji \& Greenwald, 2002; Richeson \& Ambady, 2003). Eine weitere Studie ergab in Bezug auf die implizite Präferenz der Eigengruppe, dass diese bei African Americans im Alter von fünf bis zwölf Jahren im Vergleich mit einer dominanten Outgroup (White Americans) nicht vorlag (Baron \& Banaji, 2009). Mit Blick auf Deutschland liegen ähnliche Ergebnisse vor: Mitglieder der Eigengruppe (ohne MIG) verfügten häufig über negative Einstellungen gegenüber der Fremdgruppe (türkischer MIG: Gawronski, Geschke \& Banse, 2003). Hinsichtlich Autostereotypen, die sich auf die eigene Gruppe beziehen, ergaben verschiedene Arbeiten, dass sich Deutsche selbst zum Beispiel als pünktlich, 
ordentlich und fleißig wahrnehmen und Menschen mit MIG (z. B. Kroaten, Türken) von Deutschen hauptsächlich als familienorientiert und heimatliebend erlebt werden (z.B. Ossenberg, 2020). Ähnlich zu den Angaben der Deutschen über Türken fielen die Autostereotype der Türken aus (Baur \& Ossenberg, 2016).

Eine weitere Erklärung dafür, dass Einstellungen unterschiedlich ausfallen können, stellen Assoziationen dar. Bei der evaluativen Konditionierung werden zwei Stimuli miteinander verknüpft, wodurch sich die Valenz eines Stimulus bedingt durch die Kombination mit einem anderen Stimulus, der entweder positiv oder negativ ist, verändern kann. Die evaluative Konditionierung beschreibt, wie Einstellungen gelernt und verändert werden können. Sie läuft automatisiert und unbewusst ab und beruht auf einer evaluativen Reaktion, die positiv beziehungsweise negativ ausfallen kann und sich in Mögen oder Ablehnung äußert (De Houwer, Thomas \& Baeyens, 2001). Im Schulkontext ist die soziale Integration, die darüber Auskunft gibt, ob ein Kind gemocht wird oder statt Zuneigung Ablehnung erfährt, ein zentrales Konstrukt. Bei Kindern ab einem Alter von fünf Jahren bildet sich ein grundlegendes Verständnis über das Konzept Einsamkeit heraus, das mit Ablehnung assoziiert ist (Cassidy \& Asher, 1992). Kinder im Alter von fünf bis zehn Jahren attribuierten Einsamkeit in der Schule darauf, dass sie von Freundschaftsgruppen getrennt oder ausgeschlossen, also nicht sozial integriert, waren (Liepins \& Cline, 2011). Für Kinder mit MIG kann die soziale Integration im Klassenkontext von besonderer Bedeutung sein, da sie als das Ausmaß des Gefühls des „Angenommenseins" durch die Mitschülerinnen und Mitschüler gilt sowie das Gefühl vollwertiges Gruppenmitglied zu sein vermittelt (Rauer \& Schuck, 2003). Eine erfahrene Ablehnung kann bei dem Abgelehnten das Bewusstsein hervorbringen, dass die Ablehnung Resultat negativer impliziter Einstellungen des Gegenübers sein könnte. Gniewosz und Noack (2008) konnten für Schülerinnen und Schüler der sechsten, achten und zehnten Klasse zeigen, dass in Klassen mit positiven Beziehungen zwischen Schülerinnen und Schülern weniger intolerante explizite Einstellungen gegenüber Ausländerinnen und Ausländern bestanden. Auch ergaben weitere Studien, dass Intergruppenkontakte und -freundschaften (Eigen- und Fremdgruppe; Kontakttheorie: Allport, 1954) mit weniger negativen Einstellungen gegenüber Fremdgruppen verknüpft sein können (z.B. Davies, Tropp, Aron, Pettigrew \& Wright, 2011; van Zalk \& Kerr, 2014). Ein weiteres wichtiges Konstrukt in dem Kontext ist die wahrgenommene Diskriminierung. Sie spiegelt die Verhaltenskomponente von Einstellungen wider und bildet $a b$, inwieweit Menschen mit MIG im Allgemeinen ungerechter behandelt werden. Wenn Personen mit MIG bedingt durch ihre Herkunft Benachteiligungen erleben, kann dies zu einer verstärkten Zuwendung zu ihrer eige- nen Gruppe (Reethnisierung) führen (Uslucan \& Yalcin, 2012). Skrobanek (2007) untersuchte beispielsweise, ob Jugendliche mit türkischem MIG aufgrund von subjektiv oder kollektiv wahrgenommener Diskriminierung mit Blick auf die Integration mit einem Rückzug auf die Werte und Normen der Herkunftsgruppe reagierten. Die Analysedaten stammten aus Abschlussklassen an Hauptschulen und Hauptschulzügen von Gesamtschulen. Es kam verstärkt zu einer (Re)Ethnisierung, wenn Jugendliche zum einen sich selbst und zum anderen ihre Eigengruppe von Diskriminierung betroffen wahrnahmen (Skrobanek, 2007). Eine Studie mit Paneldaten aus Finnland, in der 293 Menschen mit MIG aus der ehemaligen Sowjetunion (Russland und Estland) untersucht wurden, konnte zeigen, dass die wahrgenommene Diskriminierung zu einer Disidentifikation mit dem Aufnahmeland und daraus folgend zu negativen Einstellungen gegenüber der Outgroup beitrug (Jasinskaja-Lahti, Liebkind \& Solheim, 2009). Anhand der Ingroup/Outgroup-Favorisierung kann für Kinder mit MIG angenommenen werden, dass ein hohes Ausmaß an wahrgenommener Diskriminierung zu positiven Einstellungen gegenüber Menschen mit MIG, der Eigengruppe, führt. Bei Kindern ohne MIG kann die wahrgenommene Diskriminierung anderer gering ausfallen, da ihnen diese Erfahrung selbst fehlt, wenn auch Einstellungen negativer Art vorhanden sein können (Brewer, 1999; Jasinskaja-Lahti et al., 2009). Diskriminierung kann die Betonung der Unterschiedlichkeit von Gruppen aufrechterhalten und zu einer positiven sozialen Identität führen (Tajfel \& Turner, 1986; Zick, 2016).

Darüber hinaus stellt die eigene Identität eine weitere Erklärungsmöglichkeit dar. Die Social Identity Theory (Tajfel \& Turner, 1986) kann auch herangezogen werden, um zu erklären, dass Menschen verschiedene soziale Identitäten, zum Beispiel in Bezug auf Ethnizität und Geschlecht haben können. In bestimmten Situationen, wie etwa Leistungssituationen in der Schule, kann die soziale Identität abgewertet werden. Bei einer starken Identifikation mit der stereotypisierten Gruppe ist eine höhere Vulnerabilität für ST gegeben und Leistungseinbußen treten auf (Good, Dweck \& Aronson, 2007). Menschen mit einem MIG entwickeln sowohl eine Identität als Mitglied ihrer Herkunftskultur als auch eine Identität bezogen auf die Aufnahmekultur (Zander \& Hannover, 2013). Demgemäß stellen die Identifikation mit der Aufnahme- und der Herkunftskultur (IAK/IHK) wichtige Aspekte dar. Bei hoher IHK werden dieser gemäß der Ingroup/Outgroup-Favorisierung als Eigengruppe positive Eigenschaften zugeschrieben (Kaiser, Hagiwara, Malahy \& Wilkins, 2009; Verkuyten, 2002). Edele, Stanat, Radmann und Segeritz (2013) konnten anhand der deutschen Ergänzungsstichprobe der PISA-Erhebung 2009 für Neuntklässlerinnen und -klässler mit MIG zeigen, dass sich $12.0 \%$ der Gesamtstichprobe stark mit 
der Aufnahmekultur und weniger stark mit der Herkunftskultur identifizierten. Die Bedeutung der IAK für die Ausprägung von Einstellungen ist noch nicht abschließend geklärt. Sie spiegelt das Zugehörigkeitsgefühl zu Deutschland wider. Bei starker IAK könnten dieser Gruppe gegenüber positivere Einstellungen bestehen (neue Eigengruppe; Aufnahmeland) im Vergleich zu der Gruppe (Herkunftsland), mit der sich weniger stark identifiziert wird (Tajfel \& Turner, 1986).

\section{Messung impliziter Einstellungen}

Die Messung von Einstellungen kann sowohl explizit über Fragebögen als auch implizit mittels Impliziter Assoziationstests (IATs) erfolgen. Aufgrund der Verzerrungstendenz bei Selbstberichten (z. B. soziale Erwünschtheit) sind implizite Maße, denen diesbezüglich eine Robustheit zugeschrieben wird, im Vorteil (Greenwald, McGhee \& Schwartz, 1998).

Der IAT misst die Assoziationsstärke in Form von Reaktionszeiten zwischen kognitiven Strukturen, so genannten Konzepten. Bei einer hohen im Vergleich zu einer niedrigen Assoziationsstärke zwischen zwei Konzepten sollte es leichter sein, die gleiche Verhaltensreaktion (z. B. das Drücken einer Taste) schneller zu zeigen (Greenwald et al., 1998; Nosek, Greenwald \& Banaji, 2007a). Die StandardIAT-Prozedur von Greenwald, Nosek und Banaji (2003) umfasst mehrere Blöcke von Diskriminationsaufgaben, die das schnelle Einsortieren von Wörtern in Zielkategorien erfordern. Der IAT wurde bereits in zahlreichen Teildisziplinen der Psychologie angewendet (z.B. Gawronski \& Conrey, 2004). Bei Erwachsenen, Jugendlichen und Kindern wurde er erfolgreich eingesetzt, um zum Beispiel Einstellungen von Lehrkräften zur Inklusion oder das Vorhandensein des Geschlechterstereotypes in Bezug auf die Domäne Mathematik bei Erstklässlerinnen und -klässlern zu untersuchen (Galdi et al., 2014; Kessels, Erbring \& Heiermann, 2014).

\section{Forschungsfragen und Hypothesen}

Aufgrund der weitreichenden Folgen negativer Einstellungen, die sich zum Beispiel im (negativen) Verhalten gegenüber Menschen mit MIG zeigen und in einem weiteren Schritt zu geringeren Schulleistungen bei Kindern mit MIG führen können (ST), gilt zu klären, ob auch schon bei Grundschulkindern in Deutschland (negative) implizite Einstellungen gegenüber Menschen mit türkischem MIG vorliegen. Es wurden folgende Forschungsfragen untersucht:
1. Liegen bei Kindern im Grundschulalter implizite Einstellungen zu Menschen mit einem türkischen MIG vor?

Hypothese 1: Basierend auf entwicklungspsychologischen Erkenntnissen wird davon ausgegangen, dass bereits im Grundschulalter negative implizite Einstellungen zu Menschen mit einem türkischen MIG vorliegen.

2. Unterscheiden sich Kinder mit türkischem und ohne MIG in den impliziten Einstellungen gegenüber Menschen mit türkischem MIG?

Hypothese 2: Vor dem Hintergrund der Ingroup/Outgroup-Favorisierung wird erwartet, dass Kinder ohne MIG negative implizite Einstellungen gegenüber Menschen mit einem türkischen MIG haben (2a). Bei Kindern mit einem türkischen MIG sollte eine Präferenz gegenüber der Eigengruppe vorliegen (2b).

3. Steht das Ausmaß der impliziten Einstellungen in $\mathrm{Zu}$ sammenhang mit sozialer Integration, wahrgenommener Diskriminierung und Identifikation mit Deutschland (IAK)?

Bezugnehmend auf die Ingroup/Outgroup-Favorisierung, die Kontakttheorie, die evaluative Konditionierung und die Social Identity Theory wurden folgende Annahmen formuliert:

Hypothese 3: Es wird angenommen, dass ein negativer Zusammenhang zwischen sozialer Integration und impliziten Einstellungen besteht. Eine als hoch wahrgenommene soziale Integration sollte mit weniger stark ausgeprägten negativen impliziten Einstellungen in Zusammenhang stehen (3a). Für die wahrgenommene Diskriminierung wird ebenfalls ein negativer Zusammenhang mit impliziten Einstellungen erwartet. Bei Kindern mit türkischem MIG, die die wahrgenommene Diskriminierung als hoch einschätzen, sollten positive implizite Einstellungen vorliegen. Bei Kindern ohne MIG, die die Diskriminierung anderer als gering wahrnehmen, sollten negative implizite Einstellungen bestehen (3b). Hinsichtlich der IAK wird ein positiver Zusammenhang angenommen. Bei Kindern, die sich stärker mit Deutschland identifizieren, sollten stärker negative implizite Einstellungen vorliegen (3c).

4. Ist bei Kindern mit türkischem MIG das Ausmaß der impliziten Einstellungen mit der IHK assoziiert?

Hypothese 4: Mit Bezug auf die Social Identity Theory und die Ingroup/Outgroup-Favorisierung wird davon ausgegangen, dass eine hohe IHK mit positiven impliziten Einstellungen zusammenhängt.

\section{Methode}

\section{Stichprobe}

Die Analysen basieren auf Angaben von 240 Kindern (44.6\% weiblich) aus 20 vierten Klassen, die im Durch- 
schnitt 9.86 Jahre $(S D=0.62)$ alt waren. Die Kinder wiesen entweder einen türkischen $(n=44)$ oder keinen MIG $(n=196)$ auf. Die Operationalisierung des MIGs erfolgte über die Familiensprache („Ich spreche zu Hause manchmal Deutsch und meistens eine andere Sprache, und zwar ." /,Ich spreche zu Hause niemals Deutsch, sondern “") ${ }^{1}$. In 14 von 20 Klassen gab es im Mittel 3.14 Kinder mit türkischem MIG. Kinder mit türkischem und ohne MIG unterschieden sich nicht in ihrem Alter und in den Halbjahreszeugnisnoten Deutsch und Mathematik als Indikatoren des schulischen Erfolgs. Unterschiede bestanden im Geschlecht und der Anzahl an Büchern im Haushalt (siehe Elektronisches Supplement [ESM] 1). Für diese beiden Variablen wurde in den Analysen kontrolliert.

\section{Instrumente}

\section{IAT}

Zur Messung der impliziten Einstellungen wurde ein IAT konzeptualisiert (Greenwald et al., 2003; Nosek et al., 2007a). Zur Erstellung des IATs und des sich anschließenden Fragebogens wurde die Open-Source-Software OpenSesame verwendet (Mathôt, Schreij \& Theeuwes, 2012). Es wurde eine OpenSesame-IAT-Vorlage genutzt, die auf die relevanten Zielkonzepte und Attribut-Zielkonzepte angepasst wurde (Pinus \& Bar-Anan, 2017).

In aufeinanderfolgenden Blöcken (siehe Abbildung 1) wurden Wörter in der Mitte eines Tablet-Bildschirms präsentiert. Die Wörter konnten zu vorgegebenen Antwortkategorien (Ziel- und Attribut-Zielkonzepte), die oben links und rechts auf dem Bildschirm angeordnet waren, durch das Drücken entsprechender Tasten zugeordnet werden (Greenwald et al., 1998; Nosek et al., 2007a). Für die Zielkonzepte „Deutsch“ und „Türkisch“ wurden die größten Städte Deutschlands und der Türkei (Statistische Ämter des Bundes und der Länder, 2019; TurkStat, 2018) sowie die beliebtesten deutschen Kindernamen aus dem Jahr 2009 und korrespondierend dazu die häufigsten türkischen Vornamen in Deutschland (Gesellschaft für deutsche Sprache, 2010, 2017) ausgewählt. Pro Kategorie lagen zehn Wörter vor. Für die Attribut-Zielkonzepte "Positiv“ und „Negativ“ wurden ebenfalls jeweils zehn schul- beziehungsweise leistungsbezogene Adjektive verwendet, die an die Skala "Good Pupil“ angelehnt waren (Tsoi \& Nicholson, 1982) und emotionale, behaviorale sowie motivationale Aspekte umfassen. Eine vollständige Liste der Wörter befindet sich im ESM 2. Zur Validierung der intendierten Kategorien wurden die Wörter vorab von zehn Expertinnen und Experten, bestehend aus Wissen-

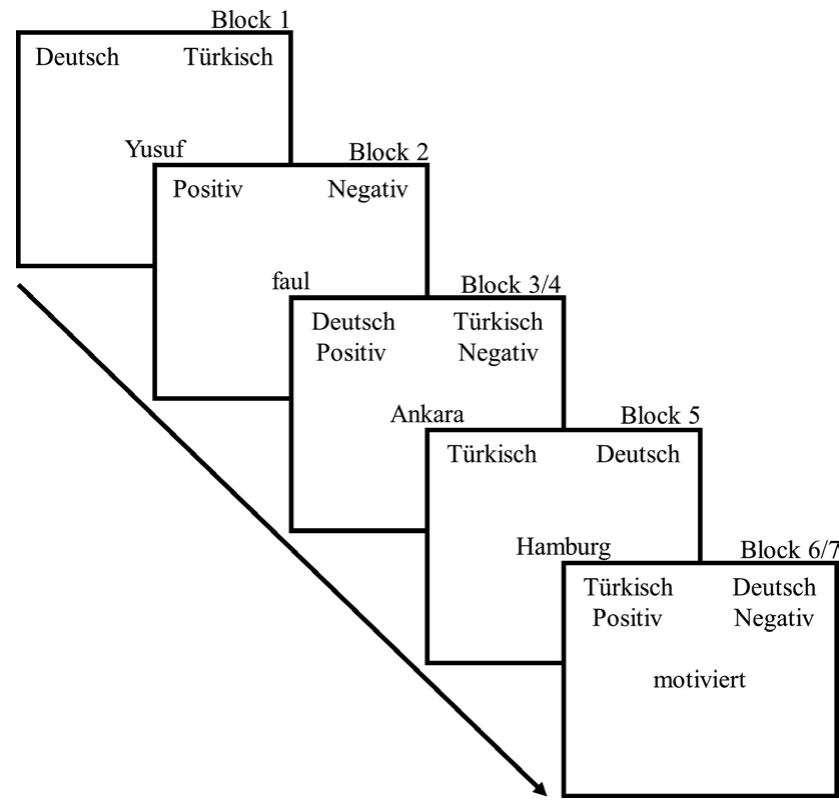

Anmerkungen: Block 1, 2, 3 und 6 umfassten je 20 Durchgänge, Block 4, 5 und 7 je 40 Durchgänge.

Abbildung 1. Vereinfachte Darstellung des Ablaufs des IATs (eigene Darstellung).

schaftlerinnen und Wissenschaftlern sowie Lehrkräften, eingruppiert. Die korrekte Zuordnung zu den Kategorien betrug jeweils $100 \%$.

\section{Soziodemografie}

Neben Alter und Geschlecht ( 0 = Junge; 1 = Mädchen) wurde die Familiensprache sowie das Geburtsland des Kindes und der Eltern $(0=$ Deutschland; 1 = Türkei) erfasst. Auch wurde erfragt, wie viele Bücher es zu Hause gab $(1=$ keine oder nur sehr wenige $(0-10$ Bücher) bis $5=$ genug, um drei oder mehr Regale zu füllen (200 Bücher); Wendt, Bos, Tarelli, Vaskova \& Walzebug, 2016).

\section{Interessierende Variablen}

Items der Sozialen Integration wurden dem Fragebogen zur Erfassung emotionaler und sozialer Schulerfahrung von Grundschulkindern dritter und vierter Klassen (FEESS 3-4; Rauer \& Schuck, 2003) entnommen. Sie geben Auskunft darüber, wie sehr sich die Kinder in der Klasse integriert fühlen (drei Items; $\alpha_{\text {Gesamtgruppe }}=.82, \alpha_{\text {türk.MIG }}=.84$, $\alpha_{\text {ohneMIG }}=.82$; z.B. „Meine Mitschülerinnen/Mitschüler sind nett zu mir."). Die Skala Diskriminierungswahrnehmung ist an die Skala der wahrgenommenen gruppenbezogenen Diskriminierung aus dem Nationalen Bildungspanel (NEPS) angelehnt und erfasst, ob die Kinder davon ausgehen, dass Menschen mit einem MIG in Deutschland dis- 
kriminiert werden (Leibniz-Institut für Bildungsverläufe e.V. [LIfBi], 2018; vier Items; $\alpha_{\text {Gesamtgruppe }}=.74, \alpha_{\text {tür.MIG }}=.66$, $\alpha_{\text {ohneMIG }}=.76$; z. B. ,Menschen, die aus einem anderen Land kommen oder eine andere Sprache sprechen, werden in Deutschland schlechter behandelt als andere."). Die Skala IAK umfasst die affektiven Items der dreidimensionalen Skala von Zander und Hannover (2013). Die Items geben Auskunft darüber, wie stark sich die Grundschulkinder mit Deutschland identifizieren und wurden so adaptiert, dass sie für Kinder dieser Altersstufe verständlicher waren (sechs Items; $\alpha_{\text {Gesamtgruppe }}=.83, \alpha_{\text {türk.MIG }}=.79, \alpha_{\text {ohnemIG }}=.82$; z. B. , Ich fühle mich stark mit den Deutschen verbunden.“). Kinder mit einem türkischen MIG beantworteten zusätzlich sechs adaptierte Items zur $I H K$, die erfassen, wie stark sie sich mit dem eigenen beziehungsweise dem Herkunftsland der Eltern verbunden fühlen (Zander \& Hannover, $2013 ; \alpha_{\text {türk.MIG }}=.81$; z.B. „Ich fühle mich stark mit diesem Land und dieser Kultur verbunden."). Alle Items wurden auf einer vierstufigen Likert-Skala gemessen $(1=$ stimmt gar nicht bis $4=$ stimmt genau). Den Tabellen 1 und 2 können die Skalenkennwerte der Gesamtstichprobe sowie der Subgruppen entnommen werden.

\section{Durchführung}

Die tabletbasierte Untersuchung wurde von geschulten Testleiterinnen und Testleitern im Klassenverband durchgeführt und umfasste zwei Schulstunden. In der ersten Stunde bearbeiteten die Kinder den IAT. Nach einer Pause beantworteten die Grundschulkinder Fragen zur Soziodemografie und zu interessierenden Variablen. Die Studienteilnahme war freiwillig und beruhte auf der Einverständniserklärung der Eltern.

Die Kinder erhielten die Anweisung, den IAT so schnell wie möglich und fehlerfrei zu bearbeiten. Fehlerhafte Antworten mussten von den Grundschulkindern selbst korri- giert werden, um fortfahren zu können. Im ersten Block bestand die Diskriminationsaufgabe darin, über die Taste „E“ und „I" die präsentierten Wörter (Hauptstädte und Vornamen) entweder in die Zielkategorie „Deutsch“ (oben links auf dem Bildschirm) oder „Türkisch“ (oben rechts auf dem Bildschirm) einzusortieren. Den Kindern wurde gesagt, dass jedes Wort nur zu einer Kategorie gehört. Im zweiten Block sollten die Adjektive in die Attribut-Zielkategorien „Positiv“ oder „Negativ“, die nun statt der Zielkonzepte „Deutsch“ und „Türkisch“ präsentiert wurden, eingeordnet werden. Pro Block wurden die zehn Zielwörter der zwei Kategorien randomisiert präsentiert. ESM 2 enthält hierzu für alle Blöcke detaillierte Angaben. Es wurde ein Interstimulusintervall von fünf Sekunden gewählt (Greenwald et al., 1998). Block 3 und 4 gelten als kompatible Bedingung, da die Zielkonzepte und AttributZielkonzepte entsprechend des negativen Stereotyps präsentiert wurden. Die Zielwörter waren entweder in die Kategorien „Deutsch“ und „Positiv“ oder in die Kategorien „Türkisch“ und „Negativ“ einzuordnen (siehe Abbildung 1). Block 3 wurde als Übung für den darauffolgen Block 4 (Test) angesehen. In Block 4 wurde die Anzahl der präsentierten Wörter von 20 auf 40 erhöht, um die Konstruktvalidität zu steigern (Nosek, Greenwald \& Banaji, 2005). In Block 5 wurde der Positionswechsel der Zielkonzepte eingeübt, sodass das Zielkonzept „Türkisch“ nun auf der linken und das Zielkonzept „Deutsch“ auf der rechten Bildschirmseite zu sehen war. Der anschließende sechste Übungs- und siebte Testblock bildete die inkompatible Bedingung. Es wurden die Kategorien „Türkisch“ und „Positiv“ sowie „Deutsch“ und „Negativ“" gepaart, die in einem Widerspruch zu dem negativen Stereotyp standen.

Die Reaktionszeiten der Blöcke 3 und 4 sowie 6 und 7 sind für die Berechnung der impliziten Einstellungen relevant (Greenwald et al., 2003; Nosek et al., 2007a). Gemäß den Berechnungskriterien wurden Reaktionszeiten über $10.000 \mathrm{~ms}$ ausgeschlossen. Fälle, bei denen die Reak-

Tabelle 1. Mittelwerte, Standardabweichungen und Interkorrelationen der Skalen und individueller Hintergrundmerkmale für die gesamte Untersuchungsstichprobe

\begin{tabular}{|c|c|c|c|c|c|c|c|c|}
\hline & & $M(S D)$ & $(2)$ & (3) & (4) & (5) & (6) & (7) \\
\hline (1) & $\mathrm{MIG}^{\mathrm{a}}$ & - & $.16^{*}$ & $-.13^{\star}$ & $-.29 *$ & -.04 & .01 & $-.21^{\star}$ \\
\hline (2) & Geschlecht $^{b}$ & - & - & .02 & $.13^{*}$ & .10 & -.02 & -.11 \\
\hline (3) & Bücher im Haushalt ${ }^{c}$ & $3.00(1.21)$ & & - & .09 & -.05 & -.02 & .04 \\
\hline (4) & $D$-Wert ${ }^{d}$ & $0.23(0.36)$ & & & - & .04 & -.10 & .13 \\
\hline (5) & Soziale Integration ${ }^{e}$ & $3.53(0.60)$ & & & & - & $-.17 \star$ & $.32 *$ \\
\hline (6) & Diskriminierung ${ }^{e}$ & $2.44(0.75)$ & & & & & - & -.11 \\
\hline (7) & Identifikation mit Deutschland ${ }^{e}$ & $3.21(0.64)$ & & & & & & - \\
\hline
\end{tabular}

Anmerkungen: $N=239$, Korrelationen basieren auf klasseninternen $z$-standardisierten Variablen; ${ }^{\mathrm{a}} 0=$ ohne MIG, $1=$ mit türkischem MIG; ${ }^{\mathrm{b}} 0=\mathrm{J}$ unge, 1 = Mädchen; ${ }^{\circ} 1$ = Keine oder nur sehr wenige (0 - 10 Bücher) bis $5=$ Genug, um drei oder mehr Regale zu füllen (200 Bücher); ${ }^{d}$ Range von -2 bis $+2 ;{ }^{e}$ Vierstufige Likert-Skala von 1 = Stimmt gar nicht bis $4=$ Stimmt genau; * $p \leq .05$. 
Tabelle 2. Mittelwerte, Standardabweichungen und Interkorrelationen der Skalen und individueller Hintergrundmerkmale getrennt für Kinder mit und ohne MIG

\begin{tabular}{|c|c|c|c|c|c|c|c|c|c|c|}
\hline & & $\begin{array}{l}\text { Mit MIG } \\
M(S D)\end{array}$ & $\begin{array}{l}\text { Ohne MIG } \\
M(S D)\end{array}$ & $(1)$ & (2) & (3) & (4) & (5) & (6) & $(7)$ \\
\hline (1) & Geschlecht $^{\mathrm{a}}$ & - & - & - & .23 & .10 & .27 & -.17 & .13 & .07 \\
\hline (2) & Bücher im Haushalt ${ }^{b}$ & $2.41(1.11)$ & $3.13(1.20)$ & -.01 & - & .12 & .26 & $-.33 *$ & .17 & .23 \\
\hline (3) & $D$-Wert ${ }^{\mathrm{C}}$ & $-0.04(0.36)$ & $0.29(0.35)$ & $.19 *$ & .03 & - & .22 & .01 & $.32 *$ & .12 \\
\hline (4) & Soziale Integration ${ }^{d}$ & $3.49(0.64)$ & $3.55(0.60)$ & .07 & -.13 & -.01 & - & -.09 & $.54^{\star}$ & $.39 *$ \\
\hline (5) & Diskriminierung ${ }^{d}$ & $2.50(0.69)$ & $2.43(0.77)$ & .02 & .05 & -.12 & $-.18^{*}$ & - & .13 & -.19 \\
\hline (6) & Identifikation mit Deutschland ${ }^{d}$ & $2.78(0.63)$ & $3.31(0.61)$ & -.12 & -.01 & .02 & $.28 *$ & $-.16 *$ & - & $.41 *$ \\
\hline (7) & $\begin{array}{l}\text { Identifikation mit der } \\
\text { Herkunftskultur }{ }^{d, e}\end{array}$ & $2.98(0.64)$ & - & - & - & - & - & - & - & - \\
\hline
\end{tabular}

Anmerkungen: Über der Diagonalen werden bivariate Pearson-Korrelationen für Kinder mit türkischem MIG berichtet ( $n=39$ ), unter der Diagonalen werden bivariate Pearson-Korrelationen für Kinder ohne MIG berichtet ( $n=195)$, Korrelationen basieren auf klasseninternen $z$-standardisierten Variablen; 0 = Junge, 1 = Mädchen; ${ }^{\text {b } 1}$ = Keine oder nur sehr wenige (0 - 10 Bücher) bis 5 = Genug, um drei oder mehr Regale zu füllen (200 Bücher); ${ }^{\circ}$ Range von -2 bis + 2; ${ }^{\circledR}$ Vier-

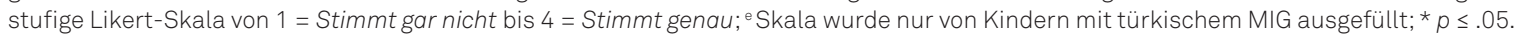

tionszeit bei mehr als $10.0 \%$ der Durchgänge $<300 \mathrm{~ms}$ betrug, lagen nicht vor. Anschließend wurden Standardabweichungen für die Blöcke 3 und 6 sowie 4 und 7 berechnet. Mittelwerte der Reaktionszeiten wurden für jeden der vier Blöcke gebildet. Als nächstes wurde die Differenz aus den mittleren Reaktionszeiten zwischen den Blöcken 6 und 3 sowie 7 und 4 ermittelt. Die Differenzen wurden durch die jeweilige Standardabweichung dividiert. Abschließend wurde der Mittelwert aus den beiden zuvor gebildeten Quotienten errechnet, der den $D$-Wert generiert und implizite Assoziationen beziehungsweise Einstellungen anzeigt. Der $D$-Wert kann Werte zwischen -2 und +2 annehmen. Ein $D$-Wert ab $|D|=0.15$ kann für das Vorliegen negativer beziehungsweise positiver Einstellungen stehen (Nosek et al., 2007b). In dieser Studie indiziert ein positiver $D$-Wert das Vorhandensein negativer impliziter Einstellungen gegenüber Menschen mit einem türkischen MIG aufgrund von sowohl kürzeren Reaktionszeiten in der stereotyp konsistenten Bedingung (Deutsch/Positiv; Türkisch/Negativ) als auch aufgrund von längeren Reaktionszeiten in der stereotyp inkonsistenten Bedingung (Deutsch / Negativ; Türkisch / Positiv). Bei einem negativen $D$-Wert liegen keine negativen impliziten Einstellungen gegenüber Menschen mit türkischem MIG vor, sondern eine Präferenz gegenüber dieser Gruppe. Ein Wert von Null indiziert, dass kein Bias vorliegt.

\section{Statistische Analysen}

Die Analysen wurden in SPSS 25 durchgeführt. Zur Beantwortung der ersten Forschungsfrage wurde das $D$-Maß berechnet (Greenwald et al., 2003), das mittels eines Einstichproben- $t$-Tests gegen \pm 0.15 getestet wurde. Eine Kovarianzanalyse mit dem $D$-Wert als abhängiger Variab- le, dem Faktor MIG (türkischer vs. ohne MIG) und den Kovariaten Geschlecht und Bücher im Haushalt wurde gerechnet, um Forschungsfrage 2 zu beantworten. (Multiple) Regressionen wurden zur Überprüfung der Forschungsfragen 3 und 4 durchgeführt. Als abhängige Variable wurde das $D$-Maß und als unabhängige Variablen soziale Integration, Diskriminierung und IAK beziehungsweise IHK aufgenommen. Bei keiner Variablen waren mehr als 1.6\% fehlende Werte zu verzeichnen (listenweiser Fallausschluss in SPSS). Ergebnisse wurden als statistisch signifikant erachtet, wenn der $p$-Wert $\leq .05$ war. Die vorliegende hierarchische Datenstruktur konnte aufgrund der Stichprobengröße in den Analysen nicht direkt berücksichtigt werden, weshalb die korrelationsbasierten Analysen anhand klasseninterner $z$-standardisierter Werte erfolgten.

\section{Ergebnisse}

\section{Deskriptive Befunde}

Anhand der deskriptiven Analysen (Tabelle 1) wird zum Beispiel für die Gesamtstichprobe ersichtlich, dass sich die Kinder im Mittel in ihren Klassen sozial integriert fühlten. Der Mittelwert wich signifikant vom theoretischen Mittel der Skala ab $(t(239)=26.45, p<.001$, $d=1.71)$. Für die Gesamtstichprobe lag ein signifikant negativer Zusammenhang zwischen MIG und der IAK vor. Kinder mit einem türkischen MIG identifizierten sich im Durchschnitt weniger mit Deutschland, was sich auch auf Mittelwertebene abzeichnete (Tabelle 2). In der Gesamt- sowie den Subgruppen gingen hohe Werte auf der Skala der sozialen Integration mit hohen Werten auf der Skala der IAK einher. 
Tabelle 3. Multiple lineare Regressionsanalysen für den Zusammenhang zwischen sozialer Integration, Diskriminierung, Identifikation mit Deutschland und impliziten Einstellungen

\begin{tabular}{|c|c|c|c|c|c|c|c|c|c|c|c|c|}
\hline \multirow{2}{*}{ Variablen } & \multicolumn{3}{|c|}{ Modell 1} & \multicolumn{3}{|c|}{ Modell 2} & \multicolumn{3}{|c|}{ Modell 3} & \multicolumn{3}{|c|}{ Modell 4} \\
\hline & $\beta$ & $S E$ & $p$ & $\beta$ & $S E$ & $p$ & $\beta$ & $S E$ & $p$ & $\beta$ & $S E$ & $p$ \\
\hline Geschlecht $^{a}$ & .15 & 0.07 & .026 & .13 & 0.07 & .041 & .13 & 0.06 & .048 & .15 & 0.06 & .021 \\
\hline Bücher im Haushalt ${ }^{b}$ & .08 & 0.06 & .242 & .08 & 0.06 & .225 & .08 & 0.06 & .203 & .07 & 0.06 & .261 \\
\hline Soziale Integration ${ }^{c}$ & -.03 & 0.07 & .691 & .02 & 0.07 & .793 & & & & & & \\
\hline Diskriminierung ${ }^{\circ}$ & -.09 & 0.07 & .188 & & & & -.10 & 0.06 & .136 & & & \\
\hline Identifikation mit Deutschland ${ }^{c}$ & .14 & 0.07 & .045 & & & & & & & .14 & 0.06 & .036 \\
\hline$R^{2}$ & & & .03 & & & .01 & & & .02 & & & .03 \\
\hline
\end{tabular}

Anmerkungen: Modell 1 und 3: $N=239$, Modelle 2 und 4: $N=240$, Berechnungen basieren auf klasseninternen z-standardisierten Variablen, Modell 1 war statistisch signifikant $\left(F(5,233)=2.47, p=.034, f^{2}=0.03\right.$, Modell 2 war statistisch nicht signifikant $\left(F(3,236)=1.98, p=.117, f^{2}=0.01\right)$, Modell $3(F(3,235)$

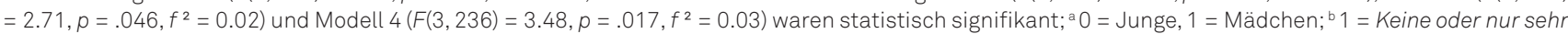
wenige (0 - 10 Bücher) bis 5 = Genug, um drei oder mehr Regale zu füllen (200 Bücher); ${ }^{c}$ Vierstufige Likert-Skala von 1 = Stimmt gar nicht bis 4 = Stimmt genau.

\section{Vorliegen impliziter Einstellungen}

In Bezug auf die erste Fragestellung zeigte sich für die Gesamtstichprobe ein mittlerer $D$-Wert in Höhe von 0.23 $(S D=0.36)$, der signifikant von 0.15 abwich $(t(239)=3.29$, $p=.001, d=0.21)$. Der Effekt ist als klein einzustufen (Cohen, 1988). Die Reaktionszeiten waren somit in der stereotyp inkonsistenten Bedingung länger als in der stereotyp konsistenten Bedingung. Es lagen negative implizite Einstellungen vor (Nosek et al., 2007b). Hypothese 1 wurde von den Daten gestützt.

\section{Unterschiede in den impliziten Einstellungen}

Im Hinblick auf die zweite Fragestellung ergab die Kovarianzanalyse einen signifikanten Haupteffekt für den Faktor MIG $\left(F(1,236)=32.98, p<.001, \eta_{\mathrm{p}}^{2}=.12\right)$. Es handelt sich um einen mittleren Effekt (Cohen, 1988). Die Kovariate Geschlecht wurde signifikant $(F(1,263)=6.77, p=.010$, $\left.\eta_{\mathrm{p}}^{2}=.03\right)$, Bücher nicht $(F(1,236)=0.86, p=.354)$. Im Mittel hatten Kinder ohne MIG negative implizite Einstellungen $\left(M_{D}=0.29, S D=0.35, t(195)=5.51, p<.001, d=0.39\right)$. Bei Kindern mit türkischem MIG lagen im Durchschnitt keine positiven impliziten Einstellungen vor $\left(M_{D}=-0.04\right.$, $S D=0.31, t(43)=2.37, p=.023, d=0.36)$. Der Mittelwert wich zudem nicht signifikant von $.00 \mathrm{ab}$, was indiziert, dass keine impliziten Einstellungen vorlagen $(t(43)=-0.88$, $p=.383, d=-0.13)$. Hypothese 2a wurde durch die Daten gestützt, $2 \mathrm{~b}$ nicht. Eine weitere Analyse, in welcher zusätzlich der Faktor Klassenkomposition (Klassen mit und ohne Kinder mit türkischem MIG) aufgenommen wurde, lieferte das gleiche Befundmuster. Es gab keinen signifikanten Effekt der Klassenkomposition (ESM 3).

\section{Zusammenhänge von sozialer Integration, Diskriminierung und Identifikation mit Deutschland mit impliziten Einstellungen}

Zur Beantwortung von Fragestellung 3 wurde eine multiple Regressionsanalyse für die Gesamtstichprobe gerechnet (Tabelle 3, Modell 1). Die IAK stand in einem positiven Zusammenhang mit negativen impliziten Einstellungen. Das bedeutet, je stärker die IAK, desto stärker negative implizite Einstellungen lagen vor. Die Höhe des Zusammenhangs ist klein. Sowohl die wahrgenommene Diskriminierung als auch die soziale Integration waren nicht statistisch signifikant mit negativen impliziten Einstellungen assoziiert. Das Geschlecht hing signifikant mit negativen impliziten Einstellungen zusammen: Mädchen hatten stärker negative implizite Einstellungen als Jungen. Bei einzelner Betrachtung der Variablen blieb das Befundmuster bestehen (Tabelle 3, Modelle 2 bis 4). Die Ergebnisse stützten Hypothese 3c, nicht jedoch 3a und 3b. In subgruppenspezifischen Analysen zeigten sich keine Zusammenhänge (ESM 3, Tabelle A). Weitere Analysen in Bezug auf die Gesamtstichprobe ergaben, dass unter Kontrolle der Klassenkomposition das Ergebnismuster bestehen blieb. Bei Aufnahme des MIGs stand die IAK nicht mehr im $\mathrm{Zu}-$ sammenhang mit impliziten Einstellungen (ESM 3, Tabellen $\mathrm{B}$ und $\mathrm{C})$.

\section{Identifikation mit der Herkunftskultur und implizite Einstellungen}

Im Hinblick auf die vierte Fragestellung wurde eine lineare Regression für die Subgruppe der Kinder mit türkischem MIG berechnet $(F(1,37)=0.55, p=.464, f=0.14)$. Die IHK stand in keinem statistisch signifikanten Zusammenhang 
mit dem negativen $D$-Wert $(\beta=.12, S E=0.16, p=.464$, $\left.R^{2}=.02\right)$. Hypothese 4 wurde nicht von den Daten gestützt.

\section{Diskussion}

In dieser Studie wurde untersucht, inwiefern Viertklässlerinnen und Viertklässler über negative implizite Einstellungen gegenüber Menschen mit einem türkischen MIG verfügen. Zusätzlich wurde analysiert, ob Unterschiede im Ausmaß der impliziten Einstellungen zwischen Kindern mit türkischem und ohne MIG bestehen und ob die soziale Integration, die wahrgenommene Diskriminierung sowie die IAK und IHK mit impliziten Einstellungen zusammenhängen.

Wie angenommen zeigte sich, dass bei Grundschulkindern negative implizite Einstellungen gegenüber Menschen mit einem türkischen MIG vorlagen. Dies steht in Einklang damit, dass Kinder ab einem Alter von zwei Jahren beginnen, implizite Einstellungen in Bezug auf Fremdgruppen (z. B. Ethnizität) zu entwickeln (Baron \& Banaji, 2006; BarTal, 1996). Gemäß unserer Annahme unterschieden sich Kinder mit türkischem und ohne MIG in ihren impliziten Einstellungen. Kinder mit türkischem MIG wiesen keine und Kinder ohne MIG wiesen negative implizite Einstellungen gegenüber Menschen mit türkischem MIG auf. Der Unterschied könnte anhand der Ingroup / Outgroup-Favorisierung erklärt werden (Brewer, 1999; Tajfel, 1982). Dass bei Kindern mit türkischem MIG keine negativen Einstellungen vorlagen, könnte auch mit einer differenzierten Kenntnis der Eigengruppe erklärt werden wie es etwa Mitglieder anderer Gruppen über die eigene Gruppe auch haben. Ebenso denkbar wäre, dass Kinder mit türkischem MIG womöglich die Stereotype kennen, jedoch diese nicht verinnerlicht haben. Entgegen unserer Hypothese lagen bei Kindern mit türkischem MIG keine positiven Einstellungen gegenüber der Eigengruppe vor. Eine Erklärung hierfür könnte die Dominanz (Status) der Fremdgruppe sein (Baron \& Banaji, 2009). Bei Kindern ohne MIG könnte das $D$ Maß auch als Reflexion subjektiv wahrgenommener Gruppenunterschiede interpretiert werden. Zur Überprüfung bedürfte es passender Kriterien (implicit stereotype accuracy: Jussim, Stevens \& Honeycutt, 2018).

Übereinstimmend mit unserer Annahme stand eine starke IAK in Zusammenhang mit negativen impliziten Einstellungen gegenüber Menschen mit türkischem MIG. Dieser Befund passt dazu, dass die Identifikation mit Deutschland (IAK), die eine Favorisierung der (neuen) Eigengruppe ermöglicht, mit negativen sozialen Kognitionen hinsichtlich der anderen Gruppe (Fremdgruppe) verknüpft sein kann (Aboud, 2003; Ingroup/Outgroup-Favorisie- rung). Entgegen unserer Annahmen zeigten sich für die soziale Integration sowie die Diskriminierung keine $\mathrm{Zu}$ sammenhänge mit Einstellungen, was an der Stärke der Assoziationen zwischen der IAK beziehungsweise Geschlecht und Einstellungen liegen könnte. Hinsichtlich des nicht signifikanten subgruppenspezifischen Ergebnisses zur IHK, das konträr zu unserer Annahme war, muss die Stichprobengröße berücksichtigt werden.

\section{Limitationen und Stärken}

Bei der Rezeption der Ergebnisse ist zu beachten, dass die geschachtelte Datenstruktur aufgrund des Stichprobenumfangs nicht im Rahmen von Mehrebenenanalysen berücksichtigt werden konnte. Um statistische Fehlschlüsse zu vermeiden, wurden klasseninterne $z$-standardisierte Werte bei den korrelationsbasierten Analysen herangezogen. Des Weiteren wurde schulisches Engagement als bedeutsames lern- und leistungsrelevantes Personenmerkmal nicht erfasst, was als Kriterium (Selbstbericht) zur Überprüfung der Stereotypengenauigkeit hätte genutzt werden können. Eine weitere Limitation ist, dass die zugrundeliegenden psychologischen Prozesse des IATs noch nicht abschließend geklärt sind (Gawronski \& Conrey, 2004). Es wird moniert, inwiefern der IAT Einstellungen misst, die bereits vor der Studiendurchführung existierten. Denkbar wäre, dass die Präsentation der Kategorien die Reaktion evoziere (Fiedler, Messner \& Bluemke, 2006). So könnten die bei Kindern ohne MIG gefundenen negativen Einstellungen Ergebnis des IATs selbst sein. Allerdings gilt der IAT als valides Messinstrument (Gawronski \& Conrey, 2004). Auch steht der Befund im Einklang mit vorherigen Arbeiten, die - z.T. in anderen Kulturen - negative Einstellungen gegenüber Fremdgruppen fanden (z.B. Steele, George, Williams \& Tay, 2018). Den Limitationen stehen Stärken gegenüber. Zur Erfassung impliziter Einstellungen wurde der IAT als non-reaktives Verfahren gewählt, sodass sozial erwünschte Antwortverzerrungen reduziert wurden. Bei sozial sensiblen Themen wie negativen Einstellungen zu Menschen mit MIG weist die implizite im Vergleich zur expliziten Messung eine deutlich höhere prädiktive Validität auf (Greenwald, Poehlman, Uhlman \& Banaji, 2009). Durch den Fokus auf Grundschulkinder und den systematischen Einbezug von für Einstellungen theoretisch relevanten Konstrukten trägt die aktuelle Studie dazu bei, den bisherigen Erkenntnisstand zu erweitern.

\section{Implikationen für Forschung und Praxis}

Die gefundenen Ergebnisse sollten repliziert und das Wissen über implizite Einstellungen bei Kindern sollte 
systematisch ausgebaut werden. Eine größere Stichprobe böte zudem die Möglichkeit, stärker auf die Klassenkomposition und die Kontakttheorie zu fokussieren. Bezugnehmend auf die weltweite Migrationsbewegung könnten weitere Migrationsgruppen in den Blick genommen werden, um Hinweise zu erhalten, inwieweit es sich um allgemeine Ingroup/Outgroup-Mechanismen oder um Einstellungen gegenüber spezifischen Migrantengruppen handelt. Mit Blick auf die Varianzaufklärung wäre es wichtig, weitere Variablen wie parentale Einstellungen zu untersuchen, da Familien zentrale Sozialisationsinstanzen sind. Im Zuge der Weitergabe von parentalen Einstellungen und Werten könnten diese mit der Ausprägung von Einstellungen zusammenhängen. Dem Zusammenhang liegt die Annahme zugrunde, dass bei Identifikation mit den Eltern, deren mögliche negative Einstellungen sich auch auf implizite Einstellungen der Kinder auswirken (Sinclair, Dunn \& Lowery, 2005). Mit Blick auf Lehrkräfte als weitere Sozialisationsinstanzen, könnten auch deren Einstellungen als Determinanten der Einstellungen von Schülerinnen und Schülern untersucht werden. In Bezug auf Geschlechterstereotype weisen bereits Studien daraufhin, dass Lehrkrafteinstellungen einerseits mit Einstellungen der Schülerinnen und Schüler korrespondieren und andererseits dazu beitragen können, dass Geschlechterunterschiede in der Leistung entwickelt werden (z.B. Carlana, 2019; Keller, 2001). Bei älteren Kindern mit türkischem MIG könnte untersucht werden, ab welchem Alter negative implizite Einstellungen gegebenenfalls verinnerlicht werden. Die Kenntnis negativer Stereotype kann ST begünstigen, weshalb die Erforschung von Bedeutung ist. Ergänzend wäre die Betrachtung der Stereotypengenauigkeit von Interesse. Neben der Übereinstimmung von Auto- und Heterostereotyp, könnte auch erforscht werden, ob die untersuchten Personen diesen Stereotypen zustimmen. Erst eine detailliertere Ursachenforschung erlaubt die Ableitung konkreter Implikationen für die Praxis (Hall \& Goh, 2017). So wäre es denkbar, Lehrkräfte für den Sachverhalt, dass bereits bei Grundschulkindern negative implizite Einstellungen vorliegen, stärker zu sensibilisieren. Des Weiteren sollten Interventionsmöglichkeiten abgeleitet werden, um negative implizite Einstellungen abzuschwächen. Wünschenswert wäre es, Programme in die alltäglichen Schulaktivitäten zu integrieren und an die spezifische Ingroup/Outgroup-Konstellation anzupassen (z. B. Förderung von Empathie und Perspektivübernahme; Beelmann \& Heinemann, 2014; Lemmer \& Wagner, 2015). Relevanz für die Praxis ergibt sich auch daraus, dass die Schule einen zentralen Sozialisationskontext darstellt, in welchem sich soziale Identitäten und Einstellungen gegenüber Gruppen entwickeln (z. B. Hannover \& Zander, 2017).

\section{Elektronische Supplemente (ESM)}

Die elektronischen Supplemente sind mit der Online-Version dieses Artikels verfügbar unter https://doi.org/ 10.1024/1010-0652/a000320

ESM 1. Ergebnisse Voranalysen

ESM 2. Zielkonzepte und Attribut-Zielkonzepte

ESM 3. Weitere Analysen

\section{Literatur}

Aboud, F. E. (2003). The formation of in-group favoritism and outgroup prejudice in young children: Are they distinct attitudes? Developmental Psychology, 39, 48 -60. https://doi.org/10.1037/ 0012-1649.39.1.48

Allport, G. W. (1954). The nature of prejudice. Cambridge, MA: Addison-Wesley.

Appel, M., Weber, S. \& Kronberger, N. (2015). The influence of stereotype threat on immigrants: Review and meta-analysis. Frontiers in Psychology, 6, 900. https://doi.org/10.3389/fpsyg.2015.00900

Asbrock, F. (2010). Stereotypes of social groups in Germany in terms of warmth and competence. Social Psychology, 41, 76 -81. https://doi.org/10.1027/1864-9335/a000011

Baron, A. \& Banaji, M. (2009). Evidence of system justification in young children. Social and Personality Psychology Compass, 3 , 918-926. https://doi.org/10.1111/j.1751-9004.2009.00214.x

Baron, A. S. \& Banaji, M. R. (2006). The development of implicit attitudes: Evidence of race evaluations from ages 6 and 10 and adulthood. Psychological Science, 17, 53 -58. https://doi.org/ 10.1111/j.1467-9280.2005.01664.x

Bar-Tal, D. (1996). Development of social categories and stereotypes in early childhood: The case of "the Arab" concept formation, stereotype and attitudes by Jewish children in Israel. International Journal of Intercultural Relations, 20,341 - 370. https:// doi.org/10.1016/0147-1767(96)00023-5

Baur, R. S. \& Ossenberg, S. (2016). Informationen zum Forschungsprojekt „Deutsche und türkische Stereotype Im Vergleich“. Universität Duisburg-Essen. Zugriff am 8. Februar 2021 unter https: //www.uni-due.de/imperia/md/images/ikk/ergebnisse_side.pdf

Beelmann, A. \& Heinemann, K. S. (2014). Preventing prejudice and improving intergroup attitudes: A meta-analysis of child and adolescent training programs. Journal of Applied Developmental Psychology, 35, 10-24. https://doi.org/10.1016/j.appdev. 2013.11.002

Bigler, R. S. \& Liben, L. S. (2006). A developmental intergroup theory of social stereotypes and prejudice. Advances in Child Development and Behavior, 34, 39 - 89. https://doi.org/10.1016/S00652407(06)80004-2

Brewer, M. B. (1999). The psychology of prejudice: Ingroup love and outgroup hate? Journal of Social Issues, 55, 429-444. https:// doi.org/10.1111/0022-4537.00126

Carlana, M. (2019). Implicit stereotypes: Evidence from teachers' gender bias. The Quarterly Journal of Economics, 134, 1163 -1224. https://doi.org/10.1093/qje/qjz008

Cassidy, J. \& Asher, S. R. (1992). Loneliness and peer relations in young children. Child Development, 63, 350-365. https://doi. org/10.1111/j.1467-8624.1992.tb01632.x

Cohen, J. (1988). Statistical power analysis for the behavioral sciences. Hillsdale: Erlbaum.

Dasgupta, N. \& Greenwald, A. G. (2001). On the malleability of automatic attitudes: Combating automatic prejudice with images of 
admired and disliked individuals. Journal of Personality and Social Psychology, 81, 800-814. https://doi.org/10.1037/00223514.81.5.800

Davies, K., Tropp, L. R., Aron, A., Pettigrew, T. F. \& Wright, S. C. (2011). Cross-group friendships and intergroup attitudes. Personality and Social Psychology Review, 15, 332-351. https://doi. org/10.1177/1088868311411103

De Houwer, J., Thomas, S. \& Baeyens, F. (2001). Associative learning of likes and dislikes: A review of 25 years of research on human evaluative conditioning. Psychological Bulletin, 127, 853-869. https://doi.org/10.1037//0033-2909.127.6.853

Dovidio, J. F., Hewstone, M., Glick, P. \& Esses, V. M. (2010). Prejudice, stereotyping and discrimination: Theoretical and empirical overview. In J. F. Dovidio, M. Hewstone, P. Glick \& V. M. Esses (Eds.), The SAGE handbook of prejudice, stereotyping and discrimination (pp.3 - 29). London: SAGE.

Eagly, A. H. \& Chaiken, S. (1995). Attitude strength, attitude structure, and resistance to change. In R. E. Petty \& J. A. Krosnick (Eds.), Attitude strength: Antecedents and consequences (pp. 413 - 433). New York, NY: Lawrence Erlbaum Associates.

Edele, A., Stanat, P., Radmann, S. \& Segeritz, M. (2013). Kulturelle Identität und Lesekompetenz von Jugendlichen aus zugewanderten Familien. In N. Jude \& E. Klieme (Hrsg.), PISA 2009 - Impulse für die Schul- und Unterrichtsforschung. 59. Beiheft der Zeitschrift für Pädagogik, 84-110.

Fazio, R. H. (1990). Multiple processes by which attitudes guide behavior: The MODE model as an integrative framework. In M. P. Zanna (Ed.), Advances in experimental social psychology (Vol. 23, pp. 75 -109). New York, NY: Academic Press.

Fiedler, K., Messner, C. \& Bluemke, M. (2006). Unresolved problems with the "I", the "A", and the "T": A logical and psychometric critique of the implicit association test (IAT). European Review of Social Psychology, 17, 74-147. https://doi.org/10.1080/104632 80600681248

Galdi, S., Cadinu, M. \& Tomasetto, C. (2014). The roots of stereotype threat: When automatic associations disrupt girls' math performance. Child Development, 85, 250-263. https://doi.org/10. $1111 /$ cdev.12128

Gawronski, B. \& Conrey, F., R. (2004). Der Implizite Assoziationstest als Maß automatischer aktivierter Assoziationen: Reichweite und Grenzen. Psychologische Rundschau, 55, 118-126. https:// doi.org/10.1026/0033-3042.55.3.118

Gawronski, B., Geschke, D. \& Banse, R. (2003). Implicit bias in impression formation: Associations influence the construal of individuating information. European Journal of Social Psychology, 33, 573 - 589. https://doi.org/10.1026/0033-3042.55.3.118

Gesellschaft für deutsche Sprache (2010). Die beliebtesten Vornamen 2009. Zugriff am 15. Dezember 2018 unter https://gfds.de/ die-beliebtesten-vornamen-2009/\#

Gesellschaft für deutsche Sprache (2017). Ausführliche Auswertung: Die beliebtesten Vornamen 2016. Zugriff am 15. Dezember 2018 unter https://gfds.de/ausfuehrliche-auswertung-diebeliebtesten-vornamen-2016/

Gilbert, D. T. \& Hixon, J. G. (1991). The trouble of thinking: Activation and application of stereotypic beliefs. Journal of Personality and Social Psychology, 60, 509-517. https://doi.org/10. 1037/0022-3514.60.4.509

Glock, S. \& Klapproth, F. (2017). Bad boys, good girls? Implicit and explicit attitudes toward ethnic minority students among elementary and secondary school teachers. Studies in Educational Evaluation, 53, 77 - 86. doi.org/10.1016/j.stueduc.2017.04.002

Gniewosz, B. \& Noack, P. (2008). Classroom climate indicators and attitudes towards foreigners. Journal of Adolescence, 31, 609 -624. https://doi.org/10.1016/j.adolescence.2007.10.006

Good, C., Dweck, C. S. \& Aronson, J. (2007). Social identity, stereotype threat, and self-theories. In A. J. Fuligni (Ed.), Contesting stereotypes and creating identities: Social categories, social identities, and educational participation (pp.115-135). New York, NY: Russell Sage Foundation.

Greenwald, A. G., McGhee, D. E. \& Schwartz, J. L. K. (1998). Measuring individual differences in implicit cognition: The Implicit Association Test. Journal of Personality and Social Psychology, 74, 1464-1480. https://doi.org/10.1037/00223514.74.6.1464

Greenwald, A. G., Nosek, B. A. \& Banaji, M. R. (2003). Understanding and using the Implicit Association Test: I. An improved scoring algorithm. Journal of Personality and Social Psychology, 85, 197 - 216. https://doi.org/10.1037/h0087889

Greenwald, A. G., Poehlman, T. A., Uhlmann, E. L. \& Banaji, M. R. (2009). Understanding and using the Implicit Association Test: III. Meta-analysis of predictive validity. Journal of Personality and Social Psychology, 97, 17-41. https://doi.org/10.1037/ a0015575

Hall, J. A. \& Goh, J. X. (2017). Studying stereotype accuracy from an integrative social-personality perspective. Social and Personality Psychology Compass, 11, e12357. https://doi.org/10.1111/ spc3.12357

Hannover, B. \& Zander, L. (2017). Einflüsse des personalen und des sozialen Selbst auf Motivation, lernrelevantes Verhalten und Bildungserträge. In J. Retelsdorf, F. Zimmermann, A. Südkamp \& O. Köller (Hrsg.), Selbstbezogene Kognitionen, sprachliche Kompetenzen und Professionalisierung von Lehrkräften (S.91 - 115). Münster:Waxmann.

Hermann, J. M. \& Vollmeyer, R. (2016). Stereotype Threat in der Grundschule. Zeitschrift für Entwicklungspsychologie und Pädagogische Psychologie, 48, 42-49. https://doi.org/10.1026/ 0049-8637/a00014

Jasinskaja-Lahti, I., Liebkind, K. \& Solheim, E. (2009). To identify or not to identify? National disidentification as an alternative reaction to perceived ethnic discrimination. Applied Psychology, 58, 105 - 128. https://doi.org/10.1111/j.1464-0597.2008.00384.x

Jussim, L. (2012). Social perception and social reality: Why accuracy dominates bias and self-fulfilling prophecy. Oxford: Oxford University Press.

Jussim, L., Crawford, J. T. \& Rubinstein, R. S. (2015). Stereotype (in) accuracy in perceptions of groups and individuals. Current Directions in Psychological Science, 24, 490 - 497. https://doi.org/ 10.1177/0963721415605257

Jussim, L., Stevens, S. T. \& Honeycutt, N. (2018). Unasked questions about stereotype accuracy. Archives of Scientific Psychology, 6, 214-229. https://doi.org/10.1037/arc0000055

Kaiser, C. R., Hagiwara, N., Malahy, L. W. \& Wilkins, C. L. (2009). Group identification moderates attitudes toward ingroup members who confront discrimination. Journal of Experimental Social Psychology, 45, 770-777. https://doi.org/10.1016/j.jesp. 2009.04.027

Keller, C. (2001). Effect of teachers' stereotyping on students' stereotyping of mathematics as a male domain. Journal of Social Psychology, 14, 165-173. https://doi.org/10.1080/00224540 109600544

Kessels, U., Erbring, S. \& Heiermann, L. (2014). Implizite Einstellungen von Lehramtstudierenden zur Inklusion. Psychologie in Erziehung und Unterricht, 61, 189-202. https://doi.org/10. 2378/peu2014.art15d

Kigel, R. M., McElvany, N. \& Becker, M. (2015). Effects of immigrant background on text comprehension, vocabulary, and reading motivation: A longitudinal study. Learning and Instruction, 35, 73 - 84. https://doi.org/10.1016/j.learninstruc.2014.10.001

Kite, M. E. \& Whitley Jr., B. E. (2016). Psychology of prejudice and discrimination (3rd Ed.). New York, NY: Routledge.

Leibniz-Institut für Bildungsverläufe e. V. (2018). Codebook. NEPS Startkohorte 2 - Kindergarten. Frühe Bildung in Kindergarten 
und Grundschule. Scientific Use File Version 7.0.0. Zugriff am 9. Juli 2019 unter https://www.neps-data.de/Portals/O/NEPS/ Datenzentrum/Forschungsdaten/SC2/7-0-0/SC2_7-0-0_ Codebook_de.pdf

Lemmer, G. \& Wagner, U. (2015). Can we really reduce ethnic prejudice outside the lab? A meta-analysis of direct and indirect contact interventions. European Journal of Social Psychology, 45, 152 - 168. https://doi.org/10.1002/ejsp.2079

Liepins, M. \& Cline, T. (2011). The development of concepts of loneliness during the early years in school. School Psychology International,32,397-411.https://doi.org/10.1177/0143034311404132

Maio, G. R., Haddock, G. \& Verplanken, B. (2019). The psychology of attitudes and attitude change. London: SAGE.

Martin, C. L. \& Ruble, D. N. (2010). Patterns of gender development. Annual Review of Psychology, 61, 353 -381. https://doi.org/10. 1146/annurev.psych.093008.100511

Martiny, S. E., Mok, S. Y., Deaux, K. \& Froehlich, L. (2014). Effects of activating negative stereotypes about Turkish-origin students on performance and identity management in German high schools. International Review of Social Psychology, 27, 205-225.

Mathôt, S., Schreij, D. \& Theeuwes, J. (2012). OpenSesame: An open-source, graphical experiment builder for the social sciences. Behavior Research Methods, 44, 314-324. https://doi. org/10.3758/s13428-011-0168-7

Nosek, B. A., Banaji, M. R. \& Greenwald, A. G. (2002). Harvesting implicit group attitudes and beliefs from a demonstration web site. Group Dynamics: Theory, Research, and Practice, 6, 101-115. https://doi.org/10.1037/1089-2699.6.1.101

Nosek, B. A., Greenwald, A. G. \& Banaji, M. R. (2005). Understanding and using the Implicit Association Test: II. Method variables and construct validity. Personality and Social Psychology Bulletin, 31,166 - 180. https://doi.org/10.1177/0146167204271418

Nosek, B. A., Greenwald, A. G. \& Banaji, M. R. (2007a). The Implicit Association Test at age 7: A methodological and conceptual review. In J. A. Bargh (Ed.), Social Psychology and the unconscious. The automaticity of higher mental processes (pp.265-292). New York, NY: Psychology Press.

Nosek, B. A., Smyth, F. L., Hansen, J. J., Devos, T., Lindner, N. M., Ranganath, K. A. et al. (2007b). Pervasiveness and correlates of implicit attitudes and stereotypes. European Review of Social Psychology, 18,1 - 53.https://doi.org/10.1080/10463280701489053

Ossenberg, S. (2020). Stereotype? Implizites Wissen und seine interkulturellen Ausprägungen in quantitativen Erhebungen. In R. Kurilla, K. Kolb-Albers, H. Krämer \& K. Pitsch (Hrsg.), Sine ira et studio: Disziplinenübergreifende Annäherung an die zwischenmenschliche Kommunikation (S.305-319). Wiesbaden: Springer.

Perugini, M. (2005). Predictive models of implicit and explicit attitudes. British Journal of Social Psychology, 44, 29 - 45. https:// doi.org/10.1348/014466604X23491

Pinus, M. \& Bar-Anan, Y. (2017). An IAT template for OpenSesame. https://doi.org/10.17605/OSF.IO/V8YAJ

Rauer, W. \& Schuck, K.-D. (2003). Fragebogen zur Erfassung emotionaler und sozialer Schulerfahrungen von Grundschulkindern dritter und vierter Klassen (FEESS 3-4). Göttingen: Beltz Test.

Richeson, J. A. \& Ambady, N. (2003). Effects of situational power on automatic racial prejudice. Journal of Experimental Social Psychology, 39, 177 - 183. https://doi.org/10.1016/S0022-1031(02) 00521-8

Rutland, A. (1999). The development of national prejudice, ingroup favouritism and self-stereotypes in British children. British Journal of Social Psychology, 38, 55-70. https://doi. org/10.1348/014466699164031

Sander, A., Ohle-Peters, A., McElvany, N., Zander, L. \& Hannover, B. (2018). Stereotypenbedrohung als Ursache für geringeren Wortschatzzuwachs bei Grundschulkindern mit Migrationshin- tergrund. Zeitschrift für Erziehungswissenschaft, 21, 177-197. https://doi.org/10.1007/s11618-017-0763-1

Schmidt-Daffy, M., Zander, L., Sander, A., McElvany, N. \& Hannover, B. (2016, März). Kompetenzbezogene Metastereotypen deutscher und türkischer Jugendlicher im Vergleich. Posterpräsentation auf der 4. Tagung der Gesellschaft für Empirische Bildungsforschung (GEBF), Berlin, Deutschland.

Sinclair, S., Dunn, E. \& Lowery, B. (2005). The relationship between parental racial attitudes and children's implicit prejudice. Journal of Experimental Social Psychology, 41, 283 - 289. https://doi. org/10.1016/j.jesp.2004.06.003

Skrobanek, J. (2007). Wahrgenommene Diskriminierung und (Re) Ethnisierung bei Jugendlichen mit türkischem Migrationshintergrund und jungen Aussiedlern. Zeitschrift für Soziologie der Erziehung und Sozialisation, 27, 265-284.

Stanat, P., Schipolowski, S., Rjosk, C., Weirich, S. \& Haag, N. (Hrsg.). (2017). IQB-Bildungstrend 2016. Kompetenzen in den Fächern Deutsch und Mathematik am Ende der 4. Jahrgangsstufe im zweiten Ländervergleich. Münster: Waxmann.

Statistische Ämter des Bundes und der Länder (2019). Die größten Städte in Deutschland nach Einwohnerzahl zum 31. Dezember 2017. Zugriff am 20. Januar 2019 unter https://de.statista.com/ statistik/daten/studie/1353/umfrage/einwohnerzahlen-dergrossstaedte-deutschlands/

Statistisches Bundesamt/Wissenschaftszentrum Berlin für Sozialforschung (2018). (Hrsg.). Datenreport 2018. Ein Sozialbericht für die Bundesrepublik Deutschland. Bonn: Bundeszentrale für politische Bildung.

Steele, C. M. \& Aronson, J. (1995). Stereotype threat and the intellectual test performance of African Americans. Journal of Personality and Social Psychology, 69, $797-811$.

Steele, J. R., George, M., Williams, A. \& Tay, E. (2018). A cross-cultural investigation of children's implicit attitudes toward White and Black racial outgroups. Developmental Science, 21, e12673. https://doi.org/10.1111/desc.12673

Tajfel, H. (1982). Social identity and intergroup relations. Cambridge: Cambridge University Press.

Tajfel, H. \& Turner, J. C. (1986). The social identity theory of intergroup behavior. In S. Worchel \& W. G. Austin (Eds.), Psychology of Intergroup Relations (pp. 7 -24). Chicago: Nelson-Hall.

Tomasetto, C., Alparone, F. R. \& Cadinu, M. (2011). Girls' math performance under stereotype threat: The moderating role of mothers' gender stereotypes. Developmental Psychology, 47, 943 - 949. https://doi.org/10.1037/a0024047

Tsoi, M. M. \& Nicholson, J. N. (1982). A factor analytic study of teachers' ratings of the personality of school children. Personality and Individual Differences, 3, 53-63. https://doi.org/10. 1016/0191-8869(82)90074-5

TurkStat. (2018). Türkei: Die größten Städte im Jahr 2019 (in Millionen Einwohner). Zugriff am 20. Januar 2019 unter https://de. statista.com/statistik/daten/studie/216185/umfrage/groesstestaedte-in-der-tuerkei/

Uslucan, H.-H. \& Yalcin, C. S. (2012). Wechselwirkungen zwischen Diskriminierung und Integration - Analyse bestehender Forschungsstände. Expertise des Zentrums für Türkeistudien und Integrationsforschung (ZfTI) im Auftrag der Antidiskriminierungsstelle des Bundes. Essen: Stiftung Zentrum für Türkeistudien und Integrationsforschung.

van Zalk, M. H. W. \& Kerr, M. (2014). Developmental trajectories of prejudice and tolerance toward immigrants from early to late adolescence. Journal of Adolescence, 43, 1658-1671. https:// doi.org/10.1007/s10964-014-0164-1

Verkuyten, M. (2002). Ethnic attitudes among minority and majority children: The role of ethnic identification, peer group victimization and parents. Social Development, 11, 558-570. https:// doi.org/10.1111/1467-9507.00215 
Wasserberg, M. J. (2014). Stereotype threat effects on African American children in an urban elementary school. The Journal of Experimental Education, 82, 502 - 517. https://doi.org/10.108 0/00220973.2013.876224

Wendt, H., Bos, W., Tarelli, I., Vaskova, A. \& Walzebug, A. (2016). IGLU/TIMSS 2011 - Skalenhandbuch zur Dokumentation der Erhebungsinstrumente und Arbeit mit den Datensätzen. Münster:Waxmann.

Zander, L. \& Hannover, B. (2013). Die Bedeutung der Identifikation mit der Herkunftskultur und mit der Aufnahmekultur Deutschland für die soziale Integration Jugendlicher mit Migrationshintergrund in ihrer Schulklasse. Zeitschrift für Entwicklungspsychologie und Pädagogische Psychologie, 45, 142-160. https:// doi.org/10.1026/0049-8637/a000092

Zander, L., Webster, G. D. \& Hannover, B. (2014). Better than me?! How adolescents with and without migration background perceive each other's performance in German classrooms. Psychology, 5, 62 -69. https://doi.org/10.4236/psych.2014.51011

Zanna, M. P. \& Rempel, J. K. (2008). Attitudes: A new look at an old concept. In R. H. Fazio \& R. E. Petty (Eds.), Key readings in social psychology. Attitudes: Their structure, function, and consequences (pp. 7 -15). New York, NY: Psychology Press.

Zick, A. (2016). Sozialpsychologische Diskriminierungsforschung. In A. Scherr, A. El-Mafaalani \& Y. E. Gökcen (Hrsg.), Handbuch Diskriminierung (S.1-22). Wiesbaden: Springer. https://doi. org/10.1007/978-3-658-11119-9_4-1

\section{Historie}

Manuskript eingereicht: 31.07.2020

Manuskript nach Revision angenommen:01.06.2021

Onlineveröffentlichung: 25.06.2021

\section{Autorenschaften}

Justine Stang und Sabrina König teilen sich die Erstautorenschaft.

\section{Förderung}

Open-Access-Veröffentlichung ermöglicht durch die Technische Universität Dortmund.

\section{ORCID}

Justine Stang

https://orcid.org/0000-0002-7177-0016

\author{
Justine Stang \\ TU Dortmund \\ Institut für Schulentwicklungsforschung \\ Vogelpothsweg 78 \\ 44227 Dortmund \\ justine.stang@tu-dortmund.de
}

